\title{
Numerical Simulation on Impacting Experiments of the Projectile Recovery System
}

\author{
Yi-Xia YAN ${ }^{\mathrm{a}}$, Gang $\mathrm{CHEN}^{\mathrm{b}}$, Zhi-Ming $\mathrm{HAO}^{\mathrm{c}}$ \\ Institute of Structural Mechanics, China Academy of Engineering Physics, Mian Yang, \\ 621900, P. R. China \\ a13990111459@163.com, bcg@caep.cn, hzm@caep.cn
}

Keywords: Recovery System, Impact Experiment, Numerical Simulation.

\begin{abstract}
The dynamic mechanical response process of the projectile impacting on the recovery system was analyzed by numerical simulation method. The deformation and failure morphology of the recovery system under the impacting experiments, including deformation data at any time during the impact experiment, were reappeared. The results of the numerical simulation were in good agreement with experimental, and provide a basis for the improvement of the structure design.
\end{abstract}

\section{Introduction}

Numerical simulation is an important way to study of impact dynamics, the finite element simulation technology research objects in the high speed collision process of stress change, compared with the experimental research, can shorten the analysis time, and reduce the cost. It involves with many degrees of freedom system, the material, and the boundary geometry, loads multiple nonlinear transient dynamic response problems, and such as solving process involves many aspects, such as: the geometric nonlinearity and contact boundary finite strain and the structural failure of treatment. In order to obtain the reliable results which are satisfied with the requirement of engineering, the initial mesh, the choice of material constitutive model, using the algorithm, the final calculation accuracy and efficiency of processing are put forward higher requirements. Projectile impacts target experiment, in order to ensure the acceleration and the ability to play a certain body better recovery, the recovery system must be reasonable designed to ensure the reliability of the impact experiment. Based on the dynamic finite element program LS-DYNA, the dynamical mechanical response process of the ball head projectile impacting recovery system of the experiment is simulated. The stress, strain and damage morphology of recycling system are obtained. By changing the structure and material of recovery system design, the weak links of recovery system are pointed out. The deformation and failure model of the recycling system obtained from the numerical simulation are consistent well with the experiment. The numerical simulation determines the experimental scheme and provides theoretical basis for structure design improved.

\section{The Experimental System Introduction}

The specimen of diameter $\Phi 120 \mathrm{~mm} \sim \Phi 230 \mathrm{~mm}$ is installed on the recovery system, through $\Phi$ $120 \mathrm{~mm}$ projectile impact, the specimen is loaded. The recovery system consists of cylinder, damping block, shielding, installation disk and the composition of connecting plate. It is $\Phi 400250$ $\mathrm{mm}$ and its quality is about $13 \mathrm{~kg}$, shown in figure 1. Its main function has two: one is the soft recovery for the projectile; Second, as a specimen holder, used to install $\Phi 120 \mathrm{~mm} \sim \Phi 230 \mathrm{~mm}$ specimen or sensors. The cylinder is collector installed base of each component parts. Equipped with diaphragm in the cylinder, cylinder can be divided into two parts. The front space is used for installation of sensors and at the back of space the damping piece is installed. The material of the cylinder is $7 \mathrm{a} 04$.And its diameter is $250 \mathrm{~mm}$, thickness is $5 \mathrm{~mm}$, shown in figure 2 . 


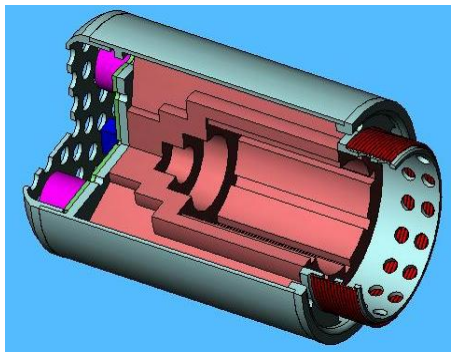

Fig. 1 Recycle System

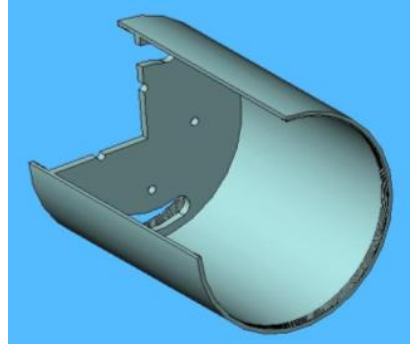

Fig.2 Cylinder

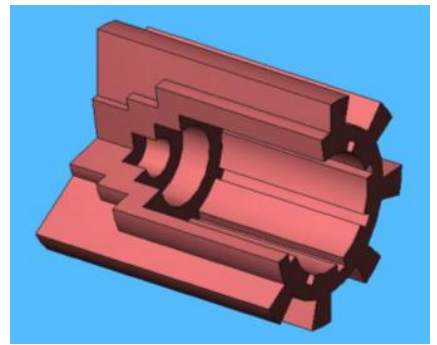

Fig. 3 Damping Block

The damping block is used to reduce and recycle the specimen. Its material is the silicon rubber. Its overall dimensions is $240 \mathrm{~mm}, 260 \mathrm{~mm}$ and the diameter of the inner hole $\mathrm{r}$ is $118 \mathrm{~mm}$, deep is $162 \mathrm{~mm}$ and quality is $6.1 \mathrm{~kg}$ (according to $1.30 \mathrm{~g} / \mathrm{cm} 3$ density gauge). To reduce the quality of the damping block, groove is in the axial direction of. Damping block is overall vulcanization molding, shown in figure 3.

The ball head projectile is adopted in the impact experiment as shown in figure 4 . The diameter of the projectile is $120 \mathrm{~mm}$, material is aluminum alloy. The impact velocity is about $150 \mathrm{~m} / \mathrm{s}$.

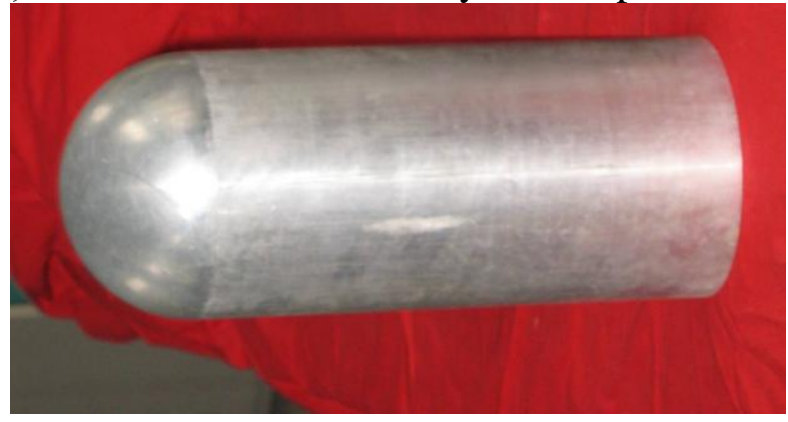

Fig. 4 Projectile of the Experiment

\section{Finite Element Simulations}

\section{Finite Element Model}

The dynamical mechanical responses process of the ball head projectile impacting to the recovery system is simulated by LS-DYNA program. The finite element model is shown in figure 5 . The numerical simulation model contains the cylinder and damping block and ignores confinement, installation disk and connecting plate.

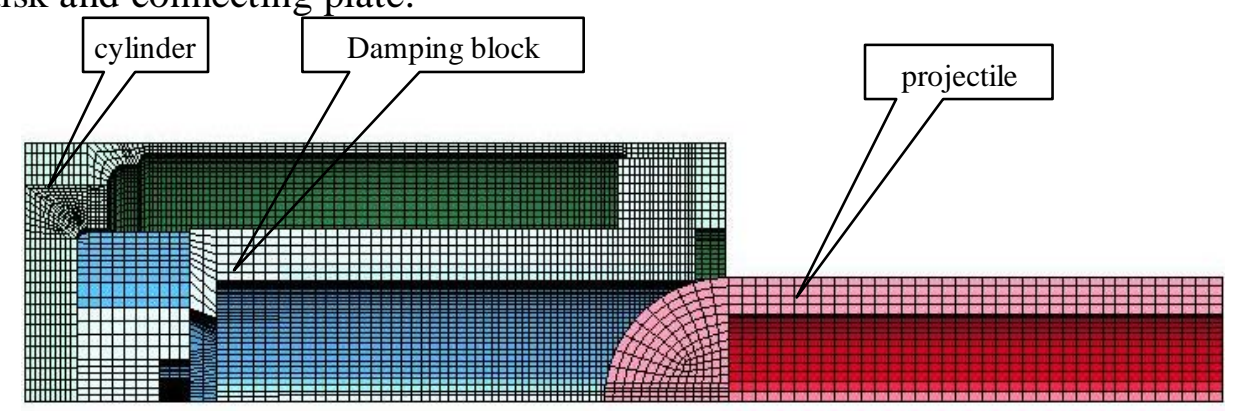

Fig. 5 The Finite Element Model of the Model I

Time integral using the explicit central difference method; Erosion dynamic contact algorithm is adopted to realize the interaction between the projectile and the recovery system. The viscous damping is used to eliminate hourglass caused by single points of integral model. In order to optimize the calculation time step and save machine, the automatic time step is adopted. 


\section{The Material Properties}

The material includes LC4, silicone rubber, LY12 and so on. An elastic-plastic model with isotropic hardening and strain rate dependence is considered in the analysis. The performance parameters are shown in table 1.

Tab.1 Material Properties

\begin{tabular}{|c|c|c|c|c|c|}
\hline & $\begin{array}{c}\text { Density } \\
\mathrm{kg} / \mathrm{m}^{3}\end{array}$ & $\begin{array}{c}\text { Elastic modulus } \\
\mathrm{MPa}\end{array}$ & $\begin{array}{c}\text { poisson's } \\
\text { ratio }\end{array}$ & $\begin{array}{c}\text { yield strength } \\
\mathrm{MPa}\end{array}$ & $\begin{array}{c}\text { tangent } \\
\text { modulus MPa }\end{array}$ \\
\hline LC4 & $2.7 \times 103$ & $70 \times 103$ & 0.3 & 390 & 5000 \\
\hline $\begin{array}{c}\text { silicone } \\
\text { rubber }\end{array}$ & $1.3 \times 103$ & 30000 & 0.3 & 2 & 8 \\
\hline LY12 & $2.78 \times 103$ & $70 \times 103$ & 0.3 & 320 & 5000 \\
\hline $45 \#$ & $7.8 \times 103$ & $210 \times 103$ & 0.3 & 360 & 21000 \\
\hline
\end{tabular}

\section{Results of the model I .}

At first, the results of model I are given. The impact velocity is $120 \mathrm{~m} / \mathrm{s}$.

The stress, strain and deformation of the damping block and cylinder are shown in Figure $6 \sim 8$. The analysis shows that the damping effect has a buffer, under the projectile impact when a large elastic deformation occurs. After impacting, there is almost no impact effect on the cylinder wall. The stress mainly concentrated at the bottom of cylinder and the largest stress occurs in the middle of the bottom. At the same time in the middle of the bottom, there is the more large plastic strain which can lead to damage. During the preliminary experiment, MiZiXing crack appeared at the bottom of cylinder as shown in figure 9 . Therefore, in order to ensure the reliability of the recovery system, it is necessary to modify the system structural design. On the one hand the damping block structure can by changed in order to let damping block absorb more energy, so as to reduce the stress of the cylinder. On the other hand it can also achieve the purpose of protect cylinder by changing the cylinder material and structure. So below context will focus on calculation results of improving model.
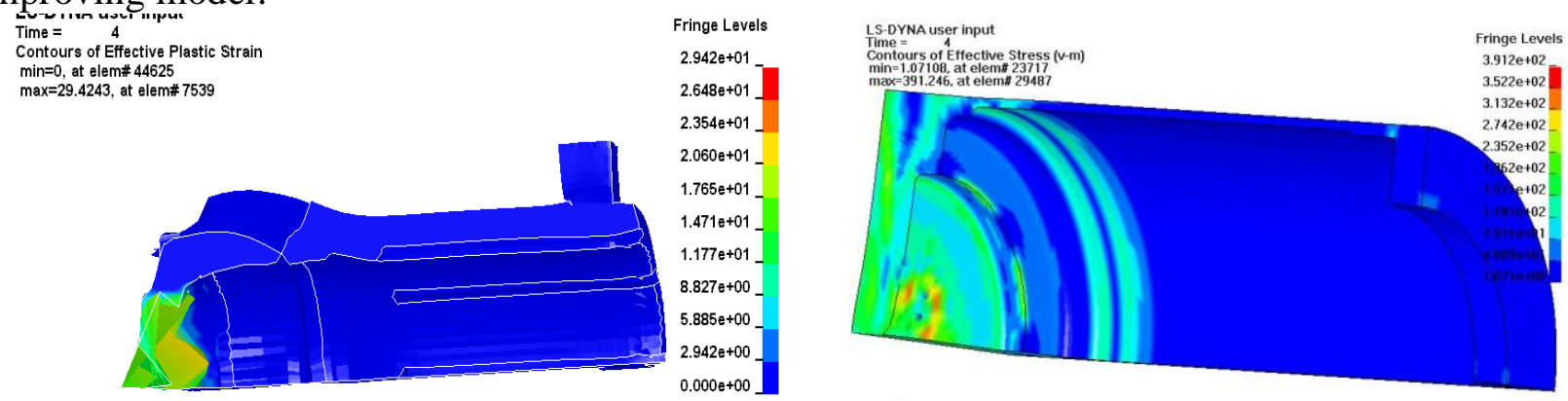

Fig. 6 Deformation of the Damping Block of the Model I Fig. 7 Equivalent Stress of the Cylinder

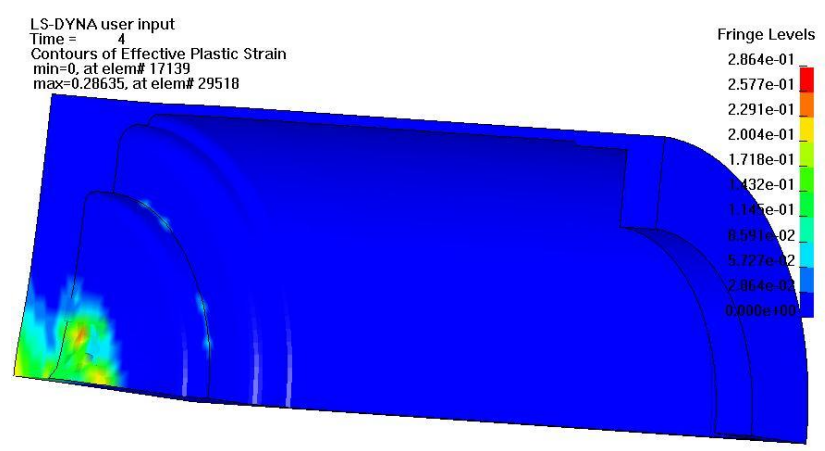

Fig. 8 Equivalent Plastic Strain of the Cylinder 


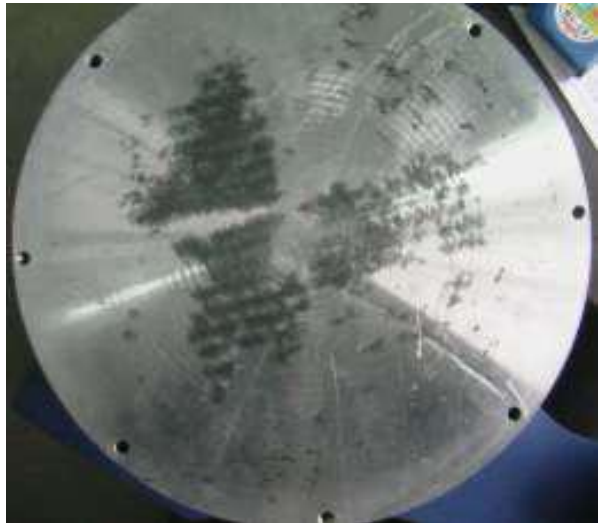

a) Backside

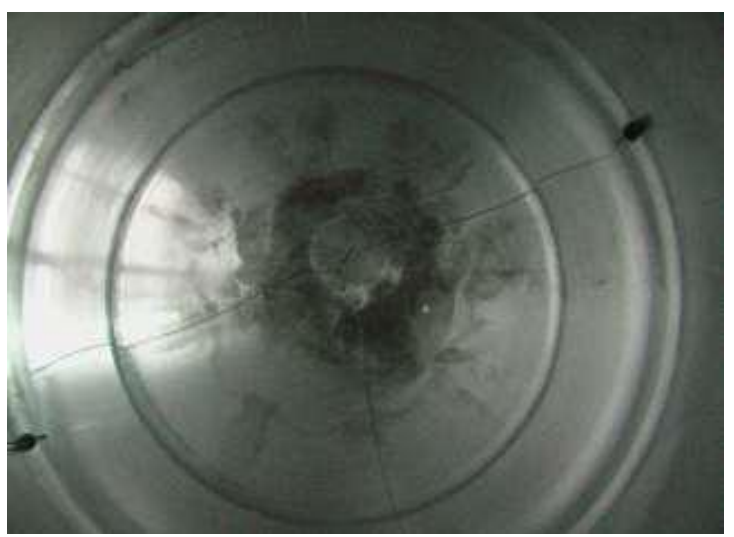

b) Face Side

Fig. 9 Deformation and Failure of the Cylinder in the Preliminary Experiment

\section{Numerical Simulation on the Improving Model}

In order to make full use of damping block buffer energy absorption effect, on the basis of the model I , damping block ear piece and steps are lengthen in the model II. At the same time, based on guarantee certain acceleration in order to avoid the cylinder bottom of the casing damage, in the middle of the cylinder bottom box punching, and change the cylinder material to 45 \#. The finite element model is shown in figure 10.

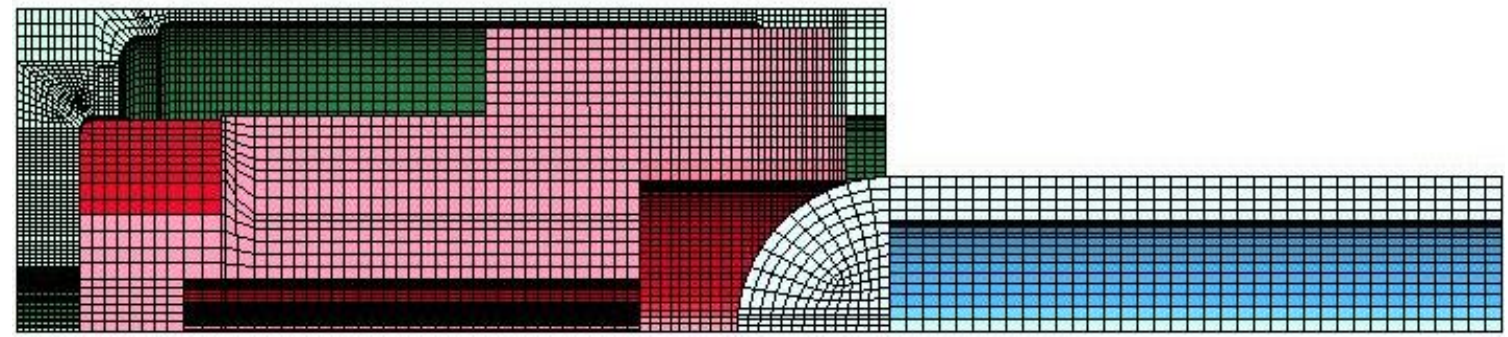

Fig. 10 Finite Element Model of the Model II

The numerical simulation and the experimental results of model II are shown in Figure $11 \sim 13$. The results show that compared with model I the deformation and failure of the model II is completely different.

Cylinder holes appear on the stress concentration at the bottom of the box, but no obvious deformation. The area of the damping block of destruction is basic equal to the size of the hole at the bottom of the cylinder. The obvious plastic deformation occurs in the front of the projectile. The calculation results reflect the experimental phenomena.

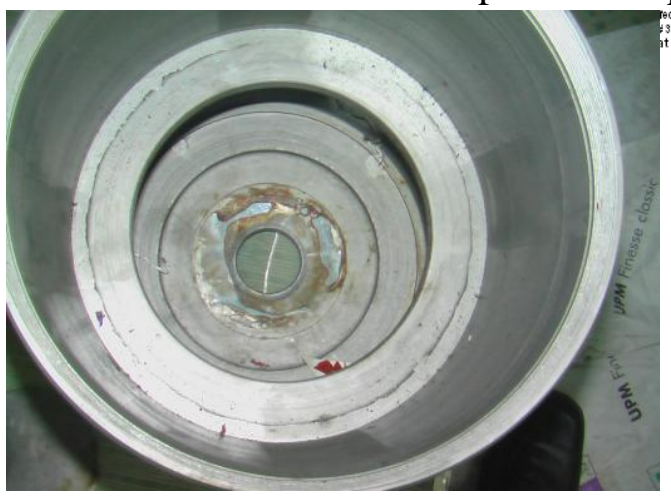

a) Calculation result

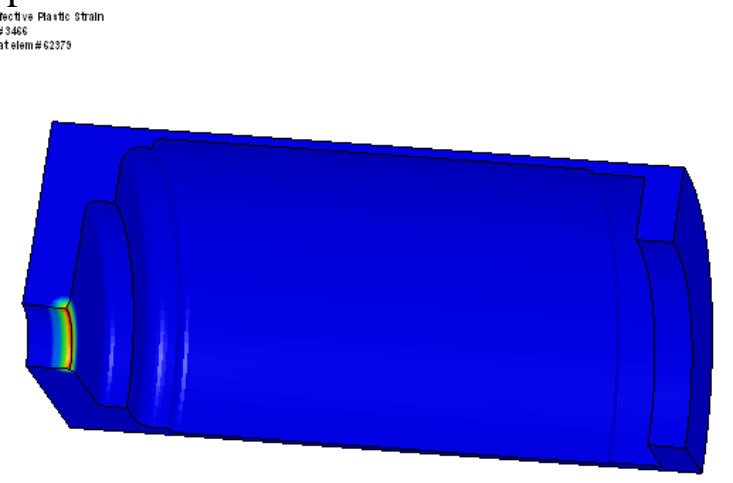

b) Experimental result

Fig. 11 Deformation of the Cylinder 


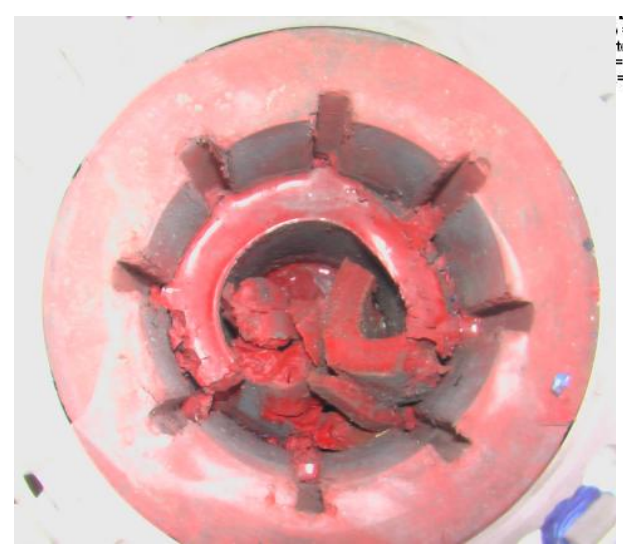

a) Calculation result

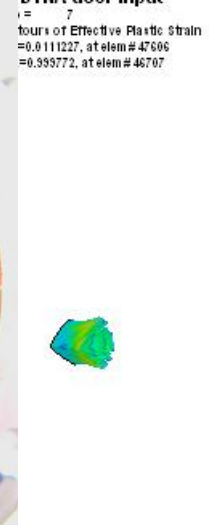

b) Experimental result

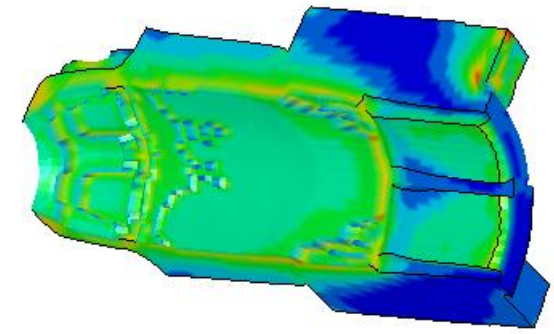

Fig. 12 Failure of the Damping Block

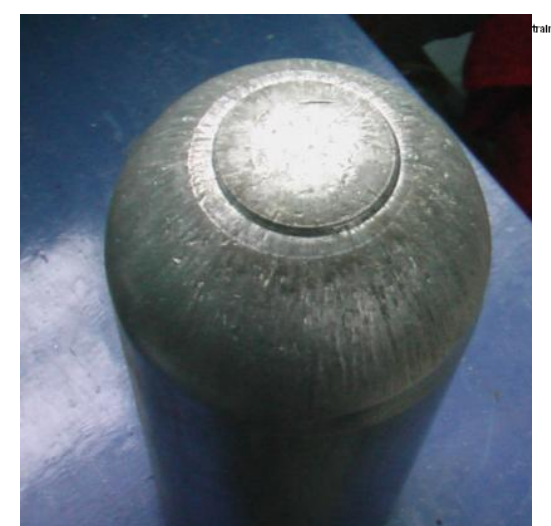

a) Calculation result

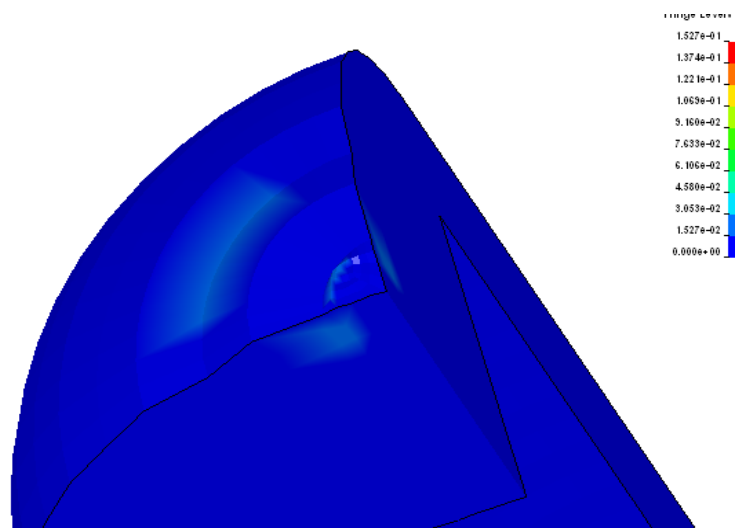

b) Experimental result

Fig.13 Deformation of the Projectile

\section{Summary}

In this paper the projectile impacting on the recovery system experiment is numerical simulated by using the finite element method.

The deformation and failure morphology of the recovery system are qualitative analysised during the ball head projectile impacting on the recovery system. The weak links of the recovery system is pointed out by the numerical simulation. The methods of improving structure are put forward, which provides a theoretical basis for improving the recovery system design and determining the scheme of the experiment.

\section{References}

[1]Chen Gang. Testing Equipment for High Force Acceleration and Broad Pulse. The Science Annual Report of CAEP. Atomic Energy Publishing House, 2012.

[2]Higgins, KE. High-G air gun provides high-level long-duration shock testing, Shock and Vibrati on Digest, Vo1.32, No.1, Jan 2000.

[3]LS-DYNA KEYWORDS USER'S MANUAL. 